OPEN ACCESS

Edited by:

Luís D. Carlos,

University of Aveiro, Portugal

Reviewed by:

Edward Gillan,

The University of lowa, United States

I. M. Dharmadasa,

Sheffield Hallam University,

United Kingdom

*Correspondence:

Katarzyna E. Hnida-Gut

khn@zurich.ibm.com

Specialty section:

This article was submitted to

Electrochemistry,

a section of the journal

Frontiers in Chemistry

Received: 06 November 2021 Accepted: 03 December 2021

Published: 20 January 2022

Citation:

Hnida-Gut KE, Sousa $M$ Hopstaken M, Reidt S, Moselund K and Schmid H (2022)

Electrodeposition as an Alternative Approach for Monolithic Integration of

InSb on Silicon.

Front. Chem. 9:810256.

doi: 10.3389/fchem.2021.810256

\section{Electrodeposition as an Alternative Approach for Monolithic Integration of InSb on Silicon}

\author{
Katarzyna E. Hnida-Gut ${ }^{1 *}$, Marilyne Sousa ${ }^{1}$, Marinus Hopstaken $^{2}$, Steffen Reidt ${ }^{1}$, \\ Kirsten Moselund ${ }^{1}$ and Heinz Schmid ${ }^{1}$
}

${ }^{1}$ IBM Research Europe-Zurich, Rüschlikon, Switzerland, ${ }^{2}$ IBM T.J. Watson Research Center-Yorktown Heights, New York, NY, United States

High-performance electronics would greatly benefit from a versatile III-V integration process on silicon. Unfortunately, integration using hetero epitaxy is hampered by polarity, lattice, and thermal expansion mismatch. This work proposes an alternative concept of III-V integration combining advantages of pulse electrodeposition, templateassisted selective epitaxy, and recrystallization from a melt. Efficient electrodeposition of nano-crystalline and stochiometric InSb in planar templates on Si (001) is achieved. The InSb deposits are analysed by high resolution scanning transmission electron microscopy (HR-STEM) and energy-dispersive X-ray spectroscopy (EDX) before and after melting and recrystallization. The results show that $\mathrm{InSb}$ can crystallise epitaxially on $\mathrm{Si}$ with the formation of stacking faults. Furthermore, X-ray photoelectron (XPS) and Auger electron (AE) spectroscopy analysis indicate that the InSb crystal size is limited by the impurity concentration resulting from the electrodeposition process.

Keywords: integration, InSb, electrodeposition, recrystallization, III-Vs, TASE

\section{INTRODUCTION}

Globally, one of the fastest-growing industries is electronic devices manufacturing based on silicon technology. With an increasing demand for faster, smaller, and better-performing devices, growth CAGR (compound annual growth rate) of $4.6 \%$ from 2020 to 2027 for this field is expected. Nonetheless, recently both academia and industry have focused efforts on the search for alternative technologies for which performance in the long run could considerably surpass that of $\mathrm{Si}$ (Ramirez et al., 2020), especially in more futuristic applications like quantum computing. These include III-V semiconductors for sensing and high-speed electronics where high value is created (Riel, 2017). The best solution would be to combine the advantages of Si and III-Vs (Hopkinson et al., 2013). However both economic and technological difficulties, like crystal lattice and polarity mismatch, have prevented the integration of foreign materials directly on a silicon platform.

One approach to address this problem, the so-called Template-Assisted Selective Epitaxy (TASE), was developed by IBM a few years ago (Schmid et al., 2015). By directing the growth by an oxide template and using metal-organic vapor phase deposition (MOCVD) to fill the designed structures, successful integration of InGaAs, InP, GaSb, etc. for high-performance MOSFETs or lasers on silicon was possible. However, MOCVD growth is limited by its low growth rates, difficulty of filling high aspect ratio structures, and the use of toxic precursors. Furthermore, not every III-V semiconductor is well suited for this technique. Examples for challenging materials include aluminum containing compounds due to the high sticking coefficient of $\mathrm{Al}$ on the mask material and antimonides due to 


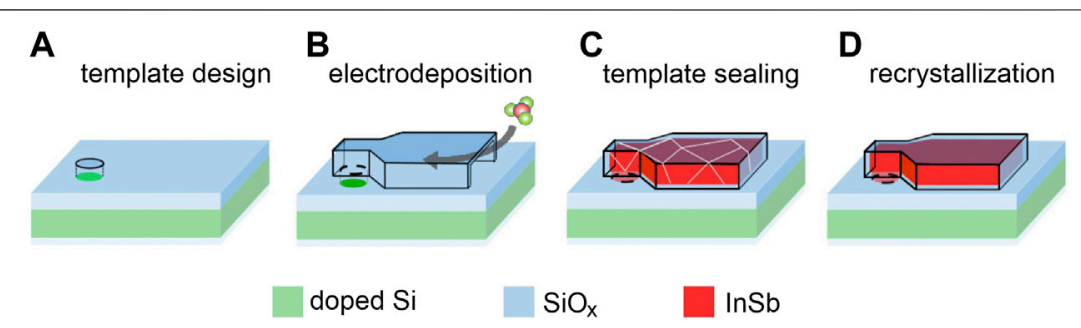

FIGURE 1 | Proposed process flow for InSb integration on Si. Conductive Si substrate with surrounding $\mathrm{SiO}_{x}$ insulation (A) Conductive Si substrate with surrounding $\mathrm{SiOx}$ insulation and (B) 3D template is immersed in the electrochemical cell. (C) After electrochemical deposition of polycrystalline InSb, the template is sealed. (D) Melting and recrystallization to form a InSb singe crystal on Si.

the small process window to supress droplet formation. InSb is a particularly interesting material with properties such as one of the smallest direct band gap $(0.17 \mathrm{eV}$ at $300 \mathrm{~K})$ and highest electron mobility $\left(77,000 \mathrm{~cm}^{2} \mathrm{~V}^{-1} \mathrm{~s}^{-1}\right)$ among III-Vs and can ensure applications in a wide range of modern devices, starting from terahertz (Liu et al., 2018a) and gamma rays detectors (Hishiki et al., 2005), through IR (Xie et al., 2016; Shi et al., 2019) and neutron-resistant Hall (Jankowski et al., 2019) sensors, ending on topological quantum devices for Majorana investigation (Chen et al., 2021). As an alternative synthesis path, electrochemical technique is considered since it was demonstrated to fill different shapes and sizes of templates efficiently, with control over composition, and using a simple set-up with water-based electrolytes (Hnida et al., 2015; Hnida et al., 2019). However, due to the characteristics of the InSb electrodeposition, the obtained material is polycrystalline, which significantly limits its practical use. To overcome this, concepts borrowed from micro zone recrystallization (Billings, 1969) and rapid melt growth (Liu et al., 2018b) can be applied. Here, the samples are subjected to a short high temperature step exceeding the melting temperature. During this step the polycrystalline sample melts and recrystallizes from a seed interface, resulting in a single crystalline structure with epitaxial relationship to the substrate.

The goal of this work is to directly integrate InSb on a silicon platform. To reach that we propose combining the three techniques, namely TASE, pulse electrodeposition, and recrystallization. The proposed process is designed to resolve the identified issues and is summarized in Figure 1. One of the key aspects is that the low melting point of InSb could allow for a CMOS compatible process.

\section{MATERIALS AND METHODS}

One of the requirements for electrodeposition of $\mathrm{InSb}$ on silicon is to provide a conductive electrode. In the presented work we used highly doped silicon wafer as global working electrode to simplify the fabrication process, while formation of addressable electrodes would require additional processing steps. Silicon wafers (001) n-type for two sets of templates having larger or smaller structures were prepared. For template fabrication, holes were etched in $100 \mathrm{~nm}$ thick $\mathrm{SiO}_{\mathrm{x}}$ on $\mathrm{Si}$ by reactive ion etching
(RIE) to define the electrode/seed area. Then, a 1,350 nm thick bis-benzocyclobutene (BCB, Dow) layer was spin coated and cross-linked at $320^{\circ} \mathrm{C}$. Subsequently, the BCB layer was patterned with the layout of template structures, etched using RIE, and coated with a $150 \mathrm{~nm}$ thick $\mathrm{SiO}_{\mathrm{x}}$ shell by plasma enhanced chemical vapor deposition (PECVD). Finally, openings were patterned on the template structures and etched into the $\mathrm{SiO}_{\mathrm{x}}$ shell exposing the BCB layer. The remaining BCB layer was etched using peroxide/sulfuric acid 1:3 solution, resulting in hollow template structures. The as-prepared substrate was dipped for $12-15 \mathrm{~s}$ in diluted $(2.5 \%) \mathrm{HF}$ to remove the native oxide on the exposed $\mathrm{Si}$ surfaces within the channels before electrodeposition.

All electrochemical syntheses were performed in a water-based electrolyte containing $0.06 \mathrm{M} \mathrm{InCl}_{3}$ (Sigma Aldrich, >98\%), $0.045 \mathrm{M} \mathrm{SbCl}_{3}$ (Sigma Aldrich, $\geq 99 \%$ ), $0.2 \mathrm{M}$ citric acid (Sigma Aldrich, $\geq 99 \%$ ), and $0.17 \mathrm{M}$ sodium citrate (Sigma Aldrich, $\geq 99 \%$ ) at room temperature. Both citric acid and sodium citrate were used as complexing agents to bring reduction potentials of indium and antimony ions closer to each other. The electrodeposition experiments were carried out using the potentiostat (BioLogic SP300) with a three-electrode setup. An $\mathrm{Ag} / \mathrm{AgCl}(3 \mathrm{M} \mathrm{NaCl})$ electrode as a reference electrode (Ref) and $\mathrm{Pt}$ wire as a counter electrode (CE) were employed. $\mathrm{SiO}_{\mathrm{x}}$ template as a working electrode (WE) was installed at a distance of $1.5 \mathrm{~cm}$ from the CE electrode. Unless otherwise stated, all potentials refer to the Ref. The fabrication of InSb nano and microstructures was performed using a cathodic pulse with $\mathrm{E}_{\text {on }}=-2.3 \mathrm{~V}$ followed by $\mathrm{E}_{\text {off }}=-0.1 \mathrm{~V}$. The potentials of "on" and "off" pulses were chosen based on Linear Sweep Voltammetry (LSV) and Open Circuit Potential (OCP) measurements on bare $n-\mathrm{Si}$, respectively. The duration of pulse "on" and "off" were 1 and $5 \mathrm{~ms}$, respectively, based on previous research (Hnida et al., 2015). During pulse "on" the reduction of In and Sb citric complexes occur, while during pulse "off" replenishment of electrolyte near the working electrode surface and dissolution of the top surface of deposit happen (Rajska et al., 2021). Section of the typical electrodeposition pulse sequence is presented in Figure 2A. Blue dotted frame marks one cycle. Slight variations in potential and current (red and green lines, respectively, slope especially visible for pulse "off") are related to the data accusation. The total deposition times $\left(\left(t_{\text {on }}+t_{\text {off }}\right) \times\right.$ number of cycles $)$ were kept in a range of 12-240 s depending on the size and shape of the 

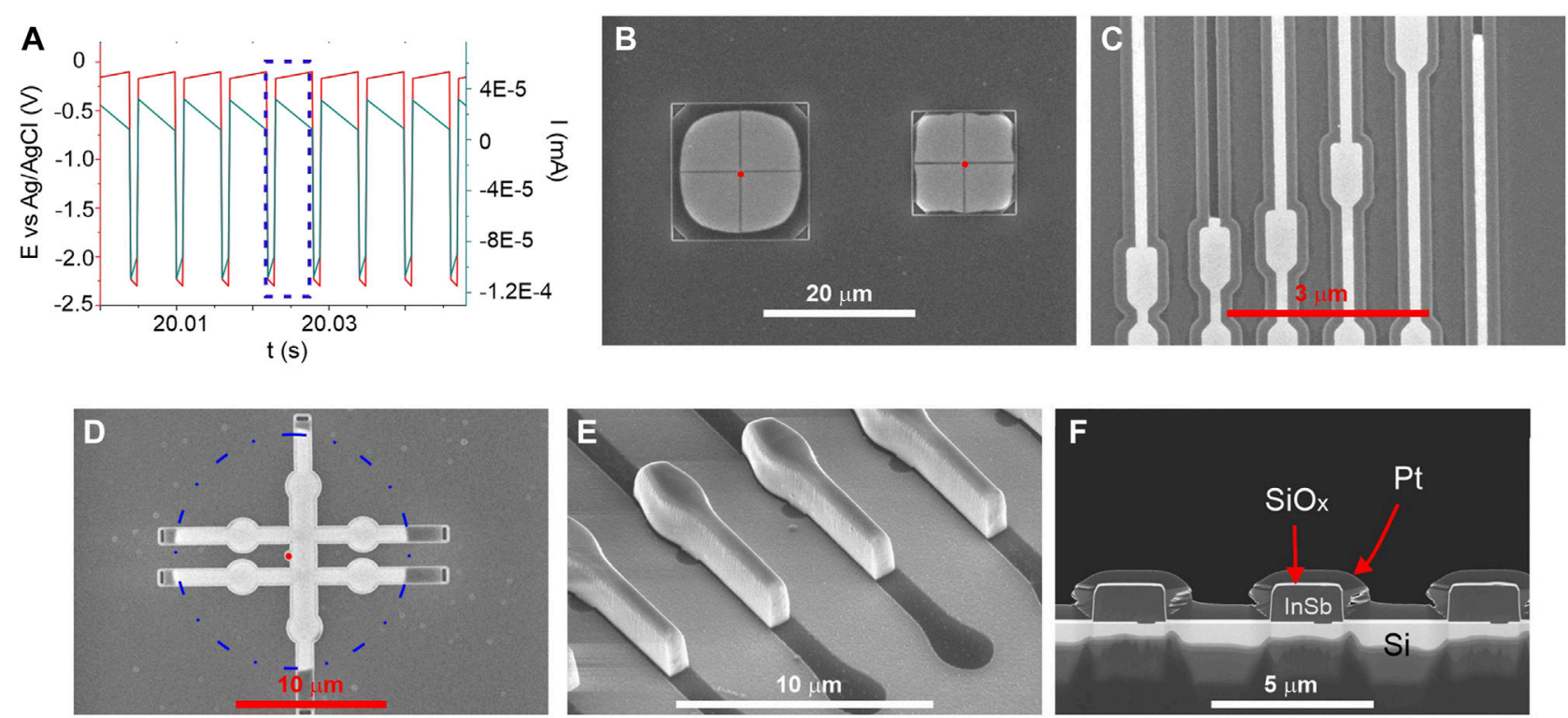

FIGURE 2 | (A) Section of typical potential pulse sequence. Blue dotted frame marks one cycle. (B-D) SEM images of filled SiO and sizes. Seed hole marked with the red dot. (E) SEM image of InSb deposit after partial removal of the SiO $\mathrm{O}_{x}$ template. The dark shade marks the area of the template which was left unfilled. (F) A cross-section of filled templates exposing the seed regions. Both $\mathrm{SiO}_{x}$ capping (thin bright part on top of the deposit) and Pt proctective (thick grey part on top of the $\mathrm{SiO}_{x}$ ) layers are marked.

oxide template and overall geometrical area of the working electrode.

For a thin intermediate InSb layer synthesis, MOCVD technique was implemented. Selective epitaxy of InSb was carried out using trimethylindium (TMIn), and trimethylantimony (TMSb) at V/III ratio 100 at $430^{\circ} \mathrm{C}$ for $65 \mathrm{~s}$, followed by $25 \mathrm{~s}$ of $\mathrm{Sb}$ deposition to further suppress the presence of In droplets.

The melting process was performed on $\mathrm{SiO}_{\mathrm{x}}$-capped samples at $535^{\circ} \mathrm{C}, 50 \mathrm{mbar}$ for $2 \mathrm{~s}$. The system was calibrated prior to the process and offset in the measured temperature was considered. After annealing, the $\mathrm{SiO}_{\mathrm{x}}$ capping layer was removed using a combination of RIE and HF etching (either wet or vapor), and the morphology, crystallinity, and chemical composition were characterized using scanning electron microscopy (SEM, Hitachi SU8000) and analytical (scanning) transmission electron microscope ((S)TEM, JEOL ARM200F) equipped with energy-dispersive X-ray spectroscopy detector. Lamellas for (S)TEM-EDX analysis were prepared using the focus ion beam technique (Dual beam FIB/SEM, FEI Helios NanoLab 450S). Priori the lamella preparation samples were covered with $250 \mathrm{~nm}$ of $\mathrm{SiO}_{\mathrm{x}}$. The samples have been measured under UHV condition with a Varian Auger system equipped with a TPIS plasma gun.

\section{RESULTS AND DISCUSSION}

In preliminary studies, we tested different silicon wafer orientations and doping levels. No change in morphology and composition between deposits was observed as long as ohmic contacts could be established. InSb deposition on BHF passivated Si showed a high nucleation density and dense film formation. However, the film adhesion was low and with the buildup of mechanical strain in the film, leading to delamination with increasing thickness already in the plating solution (HnidaGut et al., 2021). Figure 2 shows representative SEM images of InSb deposited in the template structures. Here, loss of adhesion and strain as encountered with planer films is not a limiting factor anymore. Figure 2B shows two squares composed of four quadrants each with the seed located in the center, and the template opening at the corners. The deposition process is very selective, and the InSb is deposited with high uniformity in each quarter. Templates with up to $10 \mu \mathrm{m}$ large free-standing structures were successfully filled, while larger ones collapsed due to capillary forces during the fabrication process. The smallest structures fabricated and filled (Figure 2C) have line width down to $120 \mathrm{~nm}$. Figure 2D illustrates uniform filling of the intended Hall bar structure with the seed area indicated as red point and a dashed circle as reference. Figure 2E shows a tilted view image of a sample where the $\mathrm{SiO}_{\mathrm{x}}$ was partially removed. The smooth top surface is a result of the replication of the inherently low surface roughness of the spin coated $\mathrm{BCB}$ film, while the sidewall roughness originates from the $\mathrm{BCB}$ etching process. Cross-sections from several structures are shown in Figure 2F, confirming the absence of gaps, voids, and cracks in the InSb.

After demonstrating the successful electrodeposition on InSb on $\mathrm{Si}$, and according to Figure 1, step D follows with melting and controlled recrystallization of the InSb from the seed area. First, the chemical composition is accessed before and after the melting step, followed by further chemical and structural analysis. Figure 3 shows (S)TEM cross-section images of two samples 


\section{A As-prepared}
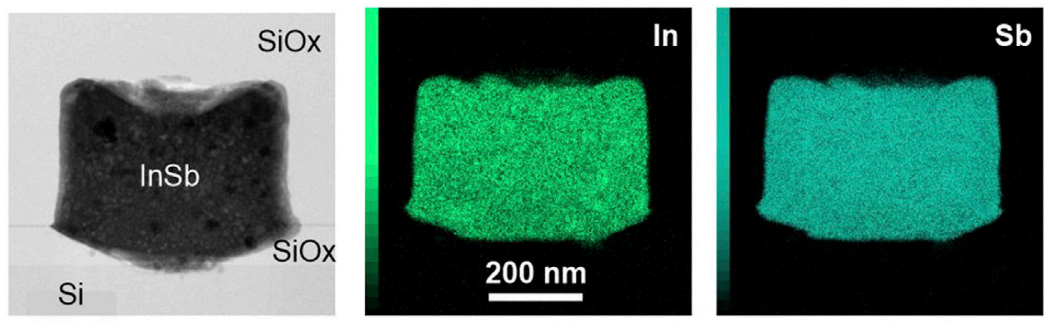

B After heat-treatement
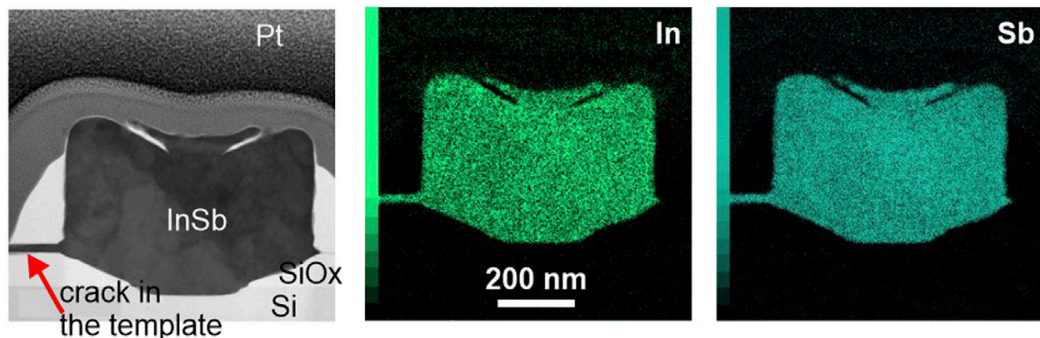

FIGURE 3 | (S)TEM images and EDX maps for (A) as-prepared and (B) annealed InSb deposits. The scale bar for all images is $200 \mathrm{~nm}$.
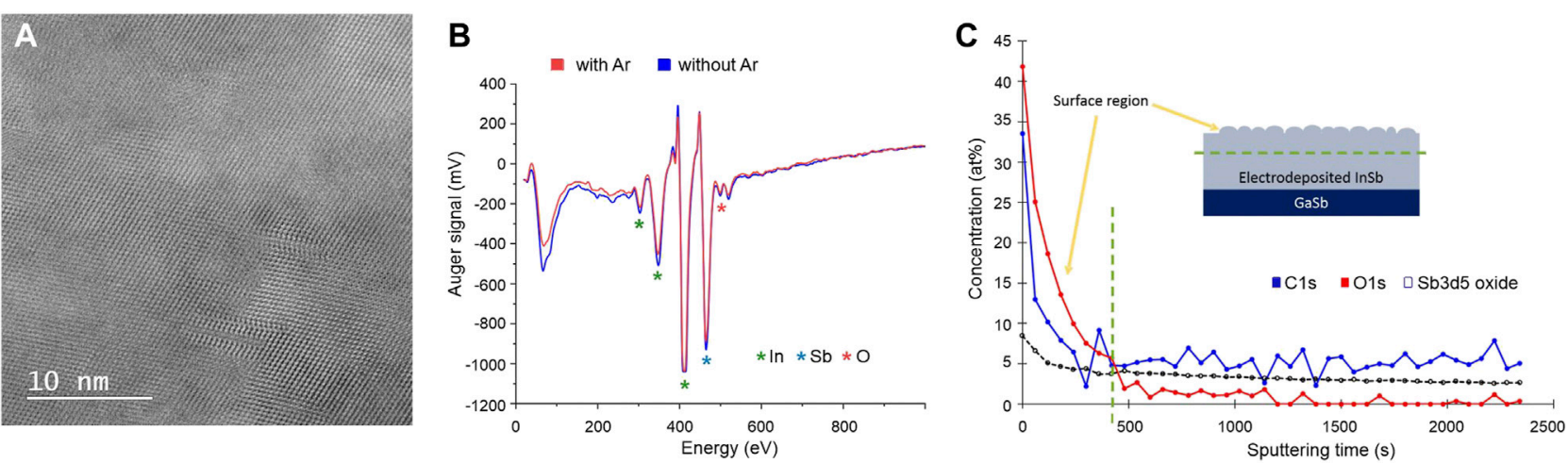

FIGURE 4 | (A) High-resolution (S)TEM image of InSb after annealing process. (B) Auger spectra for samples electrodeposited with (blue) and without (red) oxygen dissolved in the electrolyte, respectively. (C) XPS depth profile of impurities [O (red), C (blue), and Sb oxide (black)] concentration (at\%) distribution in electrodeposited InSb. Sputtering time is directly correlated with the thickness of the deposit. A green dotted line marks the surface region with indicates the presence of electrolyte residues and surface oxides.

(as-prepared and annealed, respectively) which were cut across the samples such as to expose seed areas and including the InSb-Si interface. We note that the unexpected roughness of the top surfaces stems from an artefact during the fabrication process. (S)TEM-EDX mapping was performed to investigate the distribution of In and $\mathrm{Sb}$ elements (Figure 3, center and rightside panels) in the deposited volume before and after annealing for $2 \mathrm{~s}$ at $535^{\circ} \mathrm{C}$. In both samples the distribution of In and $\mathrm{Sb}$ atoms is uniform with no detectable phase separation. The In/Sb ratio for the as-prepared sample was $45 / 55( \pm 10 \%)$ and remained constant after annealing. In Figures 3A,B a small crack is visible on the left (marked with the red arrow) which was created during the annealing procedure, caused by volume expansion of the melt or thermal stress in the oxide template. Interestingly, the resulting void is completely filled with InSb, thus confirm the melting of the deposit. After the RTA process voids in the InSb structures were frequently detected by SEM inspection, indicating loss of material. This observation was also reported in Menon et al. (2021) and needs further investigation.

Structural analysis using bright field (BF) HR-(S)TEM revealed unexpectedly that both samples remained throughout nanocrystalline with no significant differences in the size of the crystals (Figure 4A), despite the fact that the melting was indeed achieved. In addition, the anticipated recrystallization from the $\mathrm{Si}$ 


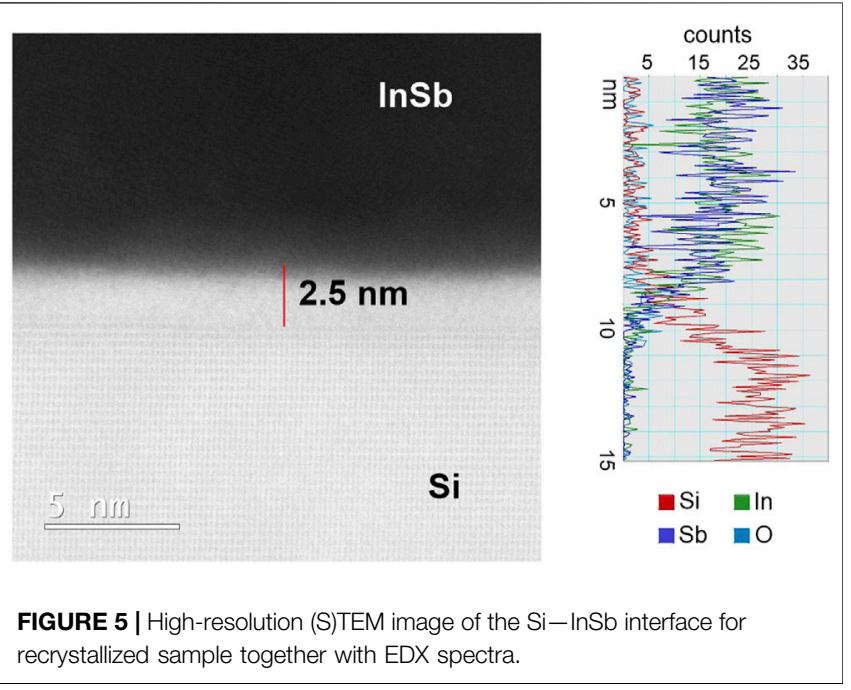

seed interface did not take place. Regarding the first observation, a possible explanation might be found in the process of diamond crystallization in metal-carbon systems. Palyanov et al. recently reported that oxygen can greatly affect the crystallization (Palyanov et al., 2020). They found that the concentration of $\mathrm{O}_{2}$ higher than $0.25 \mathrm{wt} \%$ can lead to spontaneous nucleation events which hinders crystal growth. As oxygen is present in the water-based electrolyte used for InSb electrodeposition, it is plausible that impurity induced nucleation is limiting the InSb crystallization. The common way of removing oxygen dissolved in the electrolyte is to deoxygenate it with inert gas (such as $\mathrm{N}_{2}$ or Ar) before and during the synthesis. To explore this hypothesis, two electrodeposition processes were conducted (namely with and without $\mathrm{O}_{2}$ dissolved in the electrolyte), after which samples were immediately stored in a glove box. Both samples were subjected to Auger electron spectroscopy measurement to determine the influence of $\mathrm{O}_{2}$ in the electrolyte on its content in the InSb deposits. Both spectra are presented in Figure 4B and reveal that regardless of whether the electrolyte used in the synthesis was deoxygenated or not, significant amounts of oxygen are incorporated. To further confirm the intrinsic oxygen and/or carbon contamination in electrodeposited InSb, XPS analysis was performed using a $\mathrm{GaSb}$ as substrate for $\mathrm{Sb}$ signal as reference. Due to overlapping spectra for $\mathrm{Sb}$ and $\mathrm{O}$, the exact deduction of how much oxygen is dissolved in the InSb deposit is, however, challenging. The XPS depth profile is shown in Figure $4 \mathrm{C}$ and confirms high concentrations of oxygen, carbon, and antimony oxide on the surface and in the bulk of the electrodeposited InSb. Impurity concentrations are stable around 5.2 at $\%, 0.8$ at $\%$, and 3.1 at $\%$ for $\mathrm{C}, \mathrm{O}$, and $\mathrm{Sb}$ oxide, respectively, which can explain the observed suppression of crystal growth.

As noted, the anticipated recrystallization from the Si interface is not observed, and in addition it is expected that the liquid melt should lead to a dissolution and therefore local etching of the $\mathrm{Si}$ surface which is also not observed in Figure 3B. A BF HR-(S)TEM of the interface of the annealed sample is presented in Figure $\mathbf{5}$ and reveals a $2.5 \mathrm{~nm}$ thick amorphous (barrier) layer between $\mathrm{Si}$ seed (bottom bright part) and InSb deposit (dark part), whose presence inhibits epitaxial contact between the Si and the InSb melt and correspondingly also etching of the Si surface. The composition of the barrier was further evaluated using EDX, and together with the (S)TEM data indicates the presence of Si- and metal (In, Sb) oxides. The unhindered effectiveness of the electroplating suggests that the amorpous barrier layer acts mainly as material diffusion barrier and barrier for epitaxy, but not as effective electrical insulator. Despite its $2.5 \mathrm{~nm}$ thickness, direct tunneling, and more importantly trap assisted tunneling (TAT) can be significant at $\sim 1 \mathrm{Vnm}^{-1}$ in low density oxides. In fact, electron tuneling at high cathodic voltages was previously observed during $\mathrm{Cu}$ electrodeposition on thin native silicon oxide (Arrington et al., 2008). Moreover, the barrier may contain intrinsically conductive In or $\mathrm{Sb}$ oxides. To overcome the formation of a barrier layer, which might itself be intrinsic to the specific electrodeposition process, a modified strategy is demonstrated in the following section.

To demonstrate the overall validility of the concept illustrated in Figure 1, the formation of a barrier layer during electrodeposition must be excluded. This small modification is introduced by selectively depositing a thin intermediate layer of InSb using MOCVD in the $\mathrm{Si}$ seed areas. This guarantees an oxide free InSb-Si interface which serves in a later step as interface for the electrodeposition of InSb, and the subsequent steps demonstrated above. Instead of complex templates, arrays of vias in a $\mathrm{SiO}_{\mathrm{x}}$ mask were patterned on $\mathrm{Si}(001)$ and used as a mask for MOCVD growth of small InSb crystals. The conditions of epitaxial growth were chosen to minimize the presence of In droplets on the InSb crystals, with randomly occurring In droplet still detectable (for detailed description please refer to section 2 Materials and Methods). The resulting InSb seeds crystals are shown in Figure 6A. Each hole contains at least one InSb crystal protecting the InSb-Si interface from reoxidation processes. Directly after MOCVD synthesis, the electrodeposition process was performed in which each $100 \mathrm{~nm}$-sized InSb crystallite was further overgrown by InSb, resulting in $>500 \mathrm{~nm}$ sized domes (Figure 6B). Finally, the samples were capped with $\mathrm{SiO}_{\mathrm{x}}$. The results of (S)TEM-EDX analysis of as-prepared and melted/ crystallized samples are presented in Figures 6C-F. The arrows in Figure 6C point to the crystalline InSb deposited by MOCVD (for better visibility marked with white dotted line) and to the nanocrystalline InSb deposited by ED. The surface roughness of the $\mathrm{ED}$ process at is clearly visible around the perimeter of the mushroom. The corresponding EDX map in Figure 6D confirms a uniform composition of the ED part, but also a slight In rich area in the InSb seed which might be caused by a In droplet formed during MOCVD growth. The differences of intensities are simply a consequence of the variable lamella thickness, with the stem being thinner compared to the hemisphere. As expected InSb-Si interface is flat.

After melting and crystallization, the morphology changes significantly, as visible in Figure 6F. According to the phase diagram, the melted phase gets saturated with $\mathrm{Si}$, leading to a dissolution of $\mathrm{Si}$, confirmed by the presence of the inverted pyramidal structure bound by four $\mathrm{Si}<111>$ planes below the seed region. This melt-back etching is a well documented 

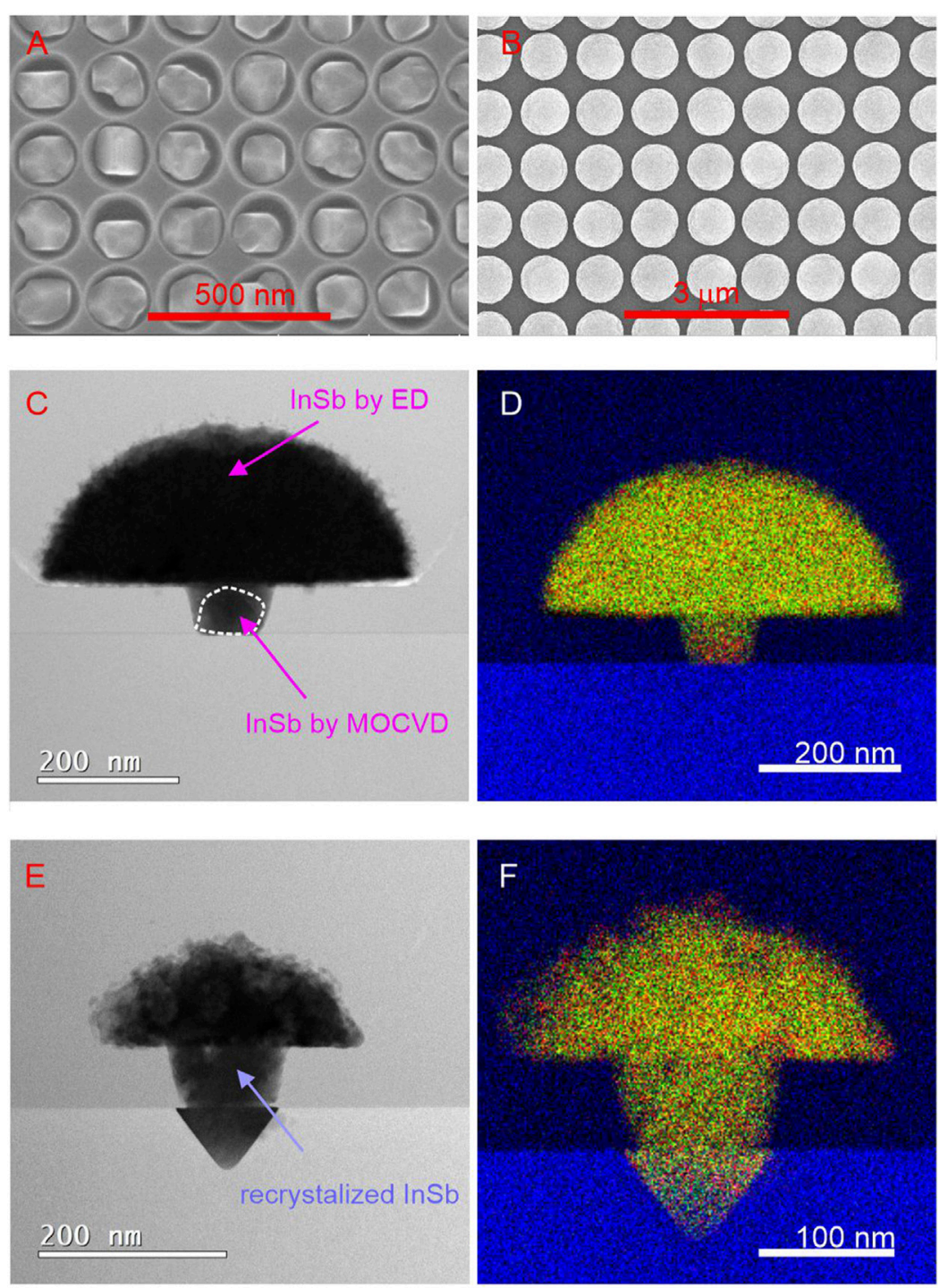

$\ln \backsim \mathrm{Sb} \square \mathrm{Si}$

FIGURE 6 | (A) SEM images of dense array of InSb crystallites by MOCVD serving as seeds for later electrodeposition. (B) sparser array similar to (A) with the small InSb seeds after the electrodeposition step resulting in large InSb mushrooms. (C) (S)TEM cross-section image of a representative structure from (B) together with (D) EDX map of In, Sb, and Si elemental distribution. (E) (S)TEM image of (B) after melting and crystallization. (F) EDX map of (E).

phenomenon and is investigated in detail for gallium (Werner et al., 2014) and gallium nitride (Khoury et al., 2017) epitaxial growth on $\mathrm{Si}$, for example. Upon cooling, the dissolved $\mathrm{Si}$ precipitates out again until reaching the equilibrium solubility limit. At first surprising is the large volume of $\mathrm{Si}$ that is meltback etched, corresponding to approximately $15 \%$ of the entire InSb volume, by several orders exceeding the solid solubility limit of $\mathrm{Si}$ in In (Olesinski et al., 1985) and Sb (Olesinski and Abbaschian, 1985). A closer look at the structure after melting and crystallisation reveals a much increased surface roughness along the perimeter and a correspondingly decreased radius of the hemisphere. This suggests that dissolved Si was diffusing through the InSb melt and precipitating out again on the opposing interface during the melt phase. The etching process slows down when the stable $<111>$ planes are formed, defined by the mask opening, but still continues at reduced speed as is noticeable by the underetching of the mask pattern. EDX measurements (Figure 6F) could not confirm the hypothesis by revealing a distinct $\mathrm{Si}$ signal at a periphery, due to the overlap of the Si signal stemming from the $\mathrm{SiO}_{\mathrm{x}}$ capping layer, and due to the likelihood of the Si forming a $\mathrm{SiO}_{\mathrm{x}}$ compound as well considering the high O level in the InSb.

Finally, structural analysis of the crystallized sample is shown in Figure 7, where an extended monocrystalline area was found (delineated by a dashed line) with the rest remaining nano crystalline. BF HR-(S)TEM revealed that this single crystalline area has the same epitaxial relationship as the $\mathrm{Si}(001)$ substrate, 

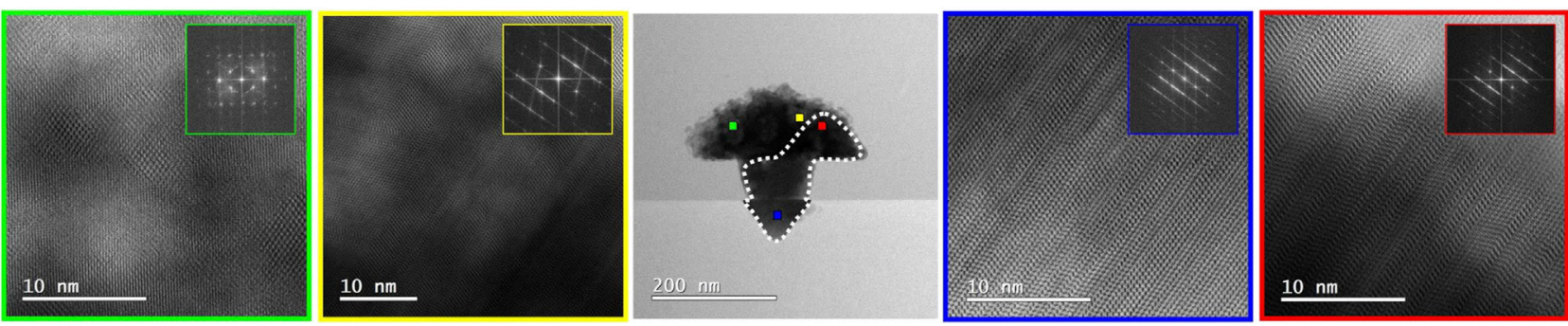

FIGURE 7 | (S)TEM analysis of the melted and crystallized InSb structure. The region with an epitaxial relationship to the substrate is marked with the white dotted line. BF HR-(S)TEM images marked with red and blue show the zinc blend monocrystalline region of the InSb structure. The remaining part of the structure is nano crystalline and marked with yellow and green.

confirming the anticipated process of initial crystallization of the melt from the Si interface. The single crystal has a high density of the stacking faults which run parallel to one of the four possible $<111>$ planes of the substrate. This is the first demonstration that the proposed concept is indeed feasible, although on a limited scale only due to the limit dictated by the impurity concentration of the InSb melt. It is worth noting that using a higher melting point semiconductor such as InAs in place of the InSb crystal deposited by MOCVD in this work, melt-back etching of the $\mathrm{Si}$ and therefore unintentional Si doping of the InSb can be avoided.

\section{CONCLUSION}

We have proposed and demonstrated the use of electrodeposition as an alternative approach for monolithic integration of a compound semiconductor on silicon. Arbritrary shaped templates were efficiently filled with nanocrystalline InSb from an aqueous solution using pulsed electrodeposition. The melted and crystallized InSb within the templates were found to remain nanocrystalline, which was ascribed to a high impurity concentration in the InSb. Complexing additives to match the reduction potentials of indium and antimony ions closer together are proposed as a source of the impurities and heterogeneous nucleation during melt cooling. Despite the limitation encountered, InSb plating and epitaxial melt crystallization from a Si surface was demonstrated on a sub-micron scale, validating the proposed integration concept. To improve the current results towards large area epitaxial InSb films on $\mathrm{Si}$, a synthesis path including brightener additives to form high purity deposits will be explored.

\section{REFERENCES}

Arrington, D., Curry, M., Street, S., Pattanaik, G., and Zangari, G. (2008). Copper Electrodeposition onto the Dendrimer-Modified Native Oxide of Silicon Substrates. Electrochimica Acta 53, 2644-2649. doi:10.1016/ j.electacta.2007.10.054

Billings, A. R. (1969). Microzone Recrystallization of Semiconductor Compound Films. J. Vacuum Sci. Tech. 6, 757-765. doi:10.1116/1.1315753

Chen, Y., Huang, S., Pan, D., Xue, J., Zhang, L., Zhao, J., et al. (2021). Strong and Tunable Spin-Orbit Interaction in a Single Crystalline InSb Nanosheet. Npj $2 d$ Mater. Appl. 5, 3. doi:10.1038/s41699-020-00184-y

\section{DATA AVAILABILITY STATEMENT}

The raw data supporting the conclusion of this article will be made available by the authors, without undue reservation.

\section{AUTHOR CONTRIBUTIONS}

$\mathrm{KH}-\mathrm{G}$ and $\mathrm{HS}$ conceived and carried out the experiments. $\mathrm{KH}-$ G, SR, and HS contributed to sample preparation. MS performed (S)TEM analysis, MH performed XPS analysis. KH-G and HS processed the experimental data, performed the analysis, drafted the manuscript, and designed the figures. KM and HS supervised the work. All authors provided critical feedback and helped shape the research, analysis, and manuscript.

\section{FUNDING}

The research was carried out thanks to the financial support of the Marie Skłodowska-Curie Action H2020 EU TECNO (894326).

\section{ACKNOWLEDGMENTS}

We thank the Cleanroom Operations Team of the Binnig and Rohrer Nanotechnology Center (BRNC) for their help and support and Andreas Bischof for performing AE spectroscopy measurements.

Hishiki, S., Kanno, I., Sugiura, O., Xiang, R., Nakamura, T., and Katagiri, M. (2005). Undoped InSb Schottky Detector for Gamma-ray Measurements. IEEE Trans. Nucl. Sci. 52, 3172-3175. doi:10.1109/TNS.2006.869817

Hnida-Gut, K. E., Sousa, M., Moselund, K. E., and Schmid, H. (2021). "Direct Electrodeposition of InSb Devices on Silicon," in Conference Proceedings of 16th IEEE Nanotechnology Materials and Devices Conference (IEEE NMDC 2021), Vancouver, Canada, 12-15.12.2021. in press.

Hnida, K. E., Bäßler, S., Akinsinde, L., Gooth, J., Nielsch, K., Socha, R. P., et al. (2015). Tuning the Polarity of Charge Transport in InSb Nanowires via Heat Treatment. Nanotechnology 26, 285701. doi:10.1088/0957-4484/26/28/285701 Hnida, K. E., Marzec, M., Wlaźlak, E., Chlebda, D., Szaciłowski, K., Gilek, D., et al. (2019). Influence of Pulse Frequency on Physicochemical Properties of InSb 
Films Obtained via Electrodeposition. Electrochimica Acta 304, 396-404. doi:10.1016/j.electacta.2019.02.111

Hopkinson, M., Martin, T., and Smowton, P. (2013). III-V Semiconductor Devices Integrated with Silicon. Semicond. Sci. Technol. 28, 090301. doi:10.1088/02681242/28/9/090301

Jankowski, J., Prokopowicz, R., Pytel, K., and El-Ahmar, S. (2019). Toward the Development of an InSb-Based Neutron-Resistant Hall Sensor. IEEE Trans. Nucl. Sci. 66, 926-931. doi:10.1109/TNS.2019.2912720

Khoury, M., Tottereau, O., Feuillet, G., Vennéguès, P., and Zúñiga-Pérez, J. (2017). Evolution and Prevention of Meltback Etching: Case Study of Semipolar GaN Growth on Patterned Silicon Substrates. J. Appl. Phys. 122, 105108. doi:10.1063/ 1.5001914

Liu, Y., Kanyang, R., Han, G., Fang, C., Zhang, J., Hao, Y., et al. (2018a). "Rainbow Trapping and Releasing in InSb Graded Grating Strip at the Terahert: Range," in 2018 Cross Strait Quad-Regional Radio Science and Wireless Technology Conference (CSQRWC), Xuzhou, China, 21-24.07.2018 (IEEE), 1-3. doi:10.1109/CSQRWC.2018.8455539

Liu, Z., Wen, J., Li, C., Xue, C., and Cheng, B. (2018b). Research Progress of Ge on Insulator Grown by Rapid Melting Growth. J. Semicond. 39, 061005. doi:10.1088/1674-4926/39/6/061005

Menon, H., Södergren, L., Athle, R., Johansson, J., Steer, M., Thayne, I., et al. (2021). Improved Quality of InSb-On-Insulator Microstructures by Flash Annealing into Melt. Nanotechnology 32, 165602. doi:10.1088/1361-6528/ abd656

Olesinski, R. W., and Abbaschian, G. J. (1985). The Sb-Si (Antimony-Silicon) System. Bull. Alloy Phase Diagrams 6, 445-448. doi:10.1007/BF02869508

Olesinski, R. W., Kanani, N., and Abbaschian, G. J. (1985). The In-Si (IndiumSilicon) System. Bull. Alloy Phase Diagrams 6, 128-130. doi:10.1007/ BF02869223

Palyanov, Y. N., Borzdov, Y. M., Kupriyanov, I. N., Bataleva, Y. V., and Nechaev, D. V. (2020). Effect of Oxygen on Diamond Crystallization in Metal-Carbon Systems. ACS Omega 5, 18376-18383. doi:10.1021/acsomega.0c02130

Rajska, D., Brzózka, A., Hnida-Gut, K. E., and Sulka, G. D. (2021). Investigation of Electrodeposition Kinetics of in, Sb, and $\mathrm{Zn}$ for Advanced Designing of InSb and ZnSb Thin Films. J. Electroanalytical Chem. 882, 114967. doi:10.1016/ j.jelechem.2020.114967

Ramirez, J. M., Malhouitre, S., Gradkowski, K., Morrissey, P. E., O’Brien, P., Caillaud, C., et al. (2020). III-V-on-Silicon Integration: From Hybrid Devices to
Heterogeneous Photonic Integrated Circuits. IEEE J. Select. Top. Quan. Electron. 26, 1-13. doi:10.1109/JSTQE.2019.2939503

Riel, H. (2017). "Integrated III-V Nanoelectronic Devices on Si," in 2017 Silicon Nanoelectronics Workshop (SNW), Kyoto, Japan, 4-5.06.2017 (IEEE), 1-2. doi:10.23919/SNW.2017.8242267

Schmid, H., Borg, M., Moselund, K., Gignac, L., Breslin, C. M., Bruley, J., et al. (2015). Template-assisted Selective Epitaxy of III-V Nanoscale Devices for Coplanar Heterogeneous Integration with Si. Appl. Phys. Lett. 106, 233101. doi:10.1063/1.4921962

Shi, C., Dong, Y., and Li, Q. (2019). High-Performance Nonequilibrium InSb PIN Infrared Photodetectors. IEEE Trans. Electron. Devices 66, 1361-1367. doi:10.1109/TED.2019.2895032

Werner, K., Beyer, A., Oelerich, J. O., Baranovskii, S. D., Stolz, W., and Volz, K. (2014). Structural Characteristics of Gallium Metal Deposited on Si (001) by MOCVD. J. Cryst. Growth 405, 102-109. doi:10.1016/j.jcrysgro.2014.07.045

Xie, C., Pusino, V., Khalid, A., Aziz, M., Steer, M. J., and Cumming, D. R. S. (2016). "Monolithic Fabrication of InSb-Based Photo-Pixel for Mid-IR Imaging," in 2016 Compound Semiconductor Week (CSW) [Includes 28th International Conference on Indium Phosphide \& Related Materials (IPRM) \& 43rd International Symposium on Compound Semiconductors (ISCS), Toyama, Japan, 26-30.06.2016 (IEEE), 1-2. doi:10.1109/ICIPRM.2016.7528581

Conflict of Interest: Authors KH-G, MS, MH, SR, KM, HS were employed by IBM Research.

Publisher's Note: All claims expressed in this article are solely those of the authors and do not necessarily represent those of their affiliated organizations, or those of the publisher, the editors, and the reviewers. Any product that may be evaluated in this article, or claim that may be made by its manufacturer, is not guaranteed or endorsed by the publisher.

Copyright $\odot 2022$ Hnida-Gut, Sousa, Hopstaken, Reidt, Moselund and Schmid. This is an open-access article distributed under the terms of the Creative Commons Attribution License (CC BY). The use, distribution or reproduction in other forums is permitted, provided the original author(s) and the copyright owner(s) are credited and that the original publication in this journal is cited, in accordance with accepted academic practice. No use, distribution or reproduction is permitted which does not comply with these terms. 